PROCEEDINGS OF THE

AMERICAN MATHEMATICAL SOCIETY

Volume 131, Number 9, Pages 2711-2713

S 0002-9939(02)06935-6

Article electronically published on December 30, 2002

\title{
ON A THEOREM OF SUPERSOLUBLE AUTOMORPHISM GROUPS
}

\author{
REZA ZOMORRODIAN
}

(Communicated by Stephen D. Smith)

\begin{abstract}
The maximal nilpotent and supersoluble automorphism groups of Riemann surfaces were given in earlier papers by this author. In this note the author wishes to correct the necessity of the condition given in Theorem (4.3) of Bounds for the order of supersoluble automorphism groups of Riemann surfaces (Proc. Amer. Math. Soc. 108 (1990), 587-600), which was left out at the time of writing the paper. The author also wishes to apologize to the readers for that.
\end{abstract}

\section{INTRODUCTION}

The main purpose of this short paper is to correct the necessity of the condition of Theorem (4.3) in 2. We also give a counterexample to that condition by introducing a new supersoluble group of order 1134 which is generated by two elements of order 2 and 3 and whose product has order 18. This group can cover a compact Riemann surface of genus 64 . We prove that it is not necessary for $g-1$ to be a power of 3 alone in order to have a supersoluble automorphism group $G$ of order $18(g-1)$ covered by a $(2,3,18)$-group. Other primes $p$ can also divide $g-1$ as long as they are all congruent to 1 modulo 3 .

Theorem ([2, Theorem (4.3)]). Let $\Gamma_{1}=\Gamma(0 ; 2,3,18)$. Let $\Omega$ be any surface of genus $g$ admitting a supersoluble automorphism group $G$ of order $18(g-1)$. Then $G$ can be covered by $\Gamma_{1}$ if and only if $g-1=3^{n} \times m$ for all $n \geq 2$, all prime divisors of $m$ being congruent to 1 modulo 3. The sufficiency of that theorem has already been proven in the same paper when $m=1$.

Proof. If $m=1$, the sufficiency of Theorem (4.3) in [2] also shows that $\Gamma_{1}$ has a supersoluble quotient group $G$ of order $2 \times 3^{n+2}$ for all $n \geq 2$ acting on a compact Riemann surface of genus $g=3^{n}+1$. Thus, $G$ has the generators $p, q$ of order 2 and 3 whose product is of order 18 .

Suppose $K=\langle z\rangle$ is a cyclic group of order $m$. Then, using the quadratic reciprocity, the congruence equation $\zeta^{2}-\zeta+1=0(\bmod m)$ has a solution $\zeta=1$ and this solution gives an automorphism $\Theta(z)=z^{l}$ of order 6 in the group $\operatorname{Aut}(K)$. By Theorem (2.7) in the same paper, the derived group $G^{\prime}$ of $G$ is nilpotent and it is easy to check that $G / G^{\prime} \cong Z_{6} \cong\langle\Theta\rangle$. Therefore, there exists a homomorphism $\Phi: G \rightarrow\langle\Theta\rangle \subseteq \operatorname{Aut}(K)$ which is defined by $\Phi(p)=\Theta^{3}, \Phi(q)=\Theta^{2}$.

Received by the editors March 15, 2002 and, in revised form, April 14, 2002.

2000 Mathematics Subject Classification. Primary 20H10; Secondary 20D15, 20D45.

(C)2002 American Mathematical Society 
We now construct a semidirect product $\Delta$ of $K$ by $G$ using the homomorphism $\Phi$ above as follows:

Let $\Delta=K \times_{\Phi} G=\{(k, g) \in K \times G \mid \Phi(g) \in \operatorname{Aut}(K)\}$, where the multiplication is the binary operation $\left(k_{1}, g_{1}\right) \times\left(k_{2}, g_{2}\right)=\left(k_{1} \Phi\left(g_{1}\right)\left(k_{2}\right), g_{1} g_{2}\right)$ for all $k_{i} \in K, g_{i} \in G$.

The elements $(1, p)$ and $(z, q)$ in $\Delta$ have order 2 and 3 , respectively, and a product of order 18. Moreover, their commutator is given by $[(1, p),(z, q)]=(z,[p, q])$. But $[p, q] \in G^{\prime}$ acts trivially on $K$ and is of order a power of 3 . Therefore, for some integer $j$ we have $[(1, p),(z, q)]^{j}=(z, 1)$. Thus, these elements generate $\Delta$. See also [3].

$K$ is cyclic and normal in $\Delta$ and $\Delta / K \cong G$ is supersoluble. Thus, by Theorem (2.4) of the same paper $\Delta=K \times_{\Phi} G=\langle(1, p),(z, q)\rangle$ is a $(2,3,18)$ supersoluble group of order $2 \times 3^{n+2} \times m$ which can cover a compact Riemann surface of genus $g=\left(3^{n} \times m\right)+1$.

Next we construct an actual example for this claim. Let $m=7$ and choose $G_{1}$ to be a $(2,3,18)$ supersoluble group of order 162 obtained in [2, page 599]. To construct a semidirect product of $G_{1}$ by $K=\left\langle z \mid z^{7}=1\right\rangle$ as above, we first find that $l=3$ is a solution of the congruence equation $\zeta^{2}-\zeta+1 \equiv 0(\bmod 7)$. Hence, $\Theta(z)=z^{3}$ is the described automorphism of order 6 in the group Aut $(K)$. We then let the action on groups be given by the equations:

$$
\Phi(p)(z)=\Theta^{3}(z)=p z p^{-1}=z^{6}, \quad \Phi(q)(z)=\Theta^{2}(z)=q z q^{-1}=z^{2} .
$$

Let $a=p z, b=q z$. It can be shown that these elements have orders 2 and 3 and a product of order 18 and generate $\Delta$. The commutator of these elements can be shown to satisfy the identity $[a, b]^{3}=z$. Therefore, a presentation for $\Delta$, i.e., a new supersoluble smooth factor group of order $|\Delta|=18(g-1)=18 \times 3^{2} \times 7=1134$ acting on a compact Riemann surface of genus $g=64$, is given as follows:

$$
\Delta=\left\langle a, b \mid a^{2}=b^{3}=(a b)^{18}=[a, b]^{21}=\left[a[a, b]^{18}, b[a, b]^{18}\right]^{3}=1 ;(a b)^{6}=(b a)^{6}\right\rangle .
$$

Using the above presentation for $\Delta$ one can also use the Magma package on the computer to check the validity of its order. Magma also gives the same answer of 1134 for the order of $\Delta$.

Remark. I must also add that although the 3-groups of automorphisms of compact Riemann surfaces take their upper bound among the (3,3,9)-groups, not all the (3,3,9)-groups are 3-groups. This fact can now be seen easily by computing the presentation for $G_{2}$ below, i.e., the only subgroup of index 2 in $\Delta$.

$$
\begin{aligned}
G_{2}=\langle c=b, d=a b a| c^{3}=d^{3}= & (c d)^{9}=\left(c d^{-1}\right)^{21} \\
& \left.=\left(c d c^{-1} d c^{-1} d^{-1} c^{-1} d\right)^{3}=1 ; \quad(c d)^{3}=(d c)^{3}\right\rangle .
\end{aligned}
$$

This is certainly not a 3 -group because its order $3^{4} \times 7=567$ is not a power of 3 , a fact which can also be checked by Magma.

\section{ACKnowledgement}

The author thanks Professor B. Srinivasan and Professor Stephen D. Smith of the University of Illinois in Chicago and apologizes to all the readers of the paper [2]. The author also thanks the referee in advance for all his criticisms and advise. 


\section{REFERENCES}

1. R. Zomorrodian, Nilpotent automorphism groups of Riemann surfaces, Trans. Amer. Math. Soc. 288 (1985), 241-255. MR 86d:20059

2. R. Zomorrodian, Bounds for the order of supersoluble automorphism groups of Riemann surfaces, Proc. Amer. Math. Soc. Vol. 108, No. 3, March (1990), 587-600. MR 90e:20045

3. C. Maclachlan and G. Gromadzki, Supersoluble groups of automorphisms of compact Riemann surfaces, Glasgow Math. J. 31 (1989), 321-327.

Department of Mathematics, University of Pittsburgh, Pittsburgh, Pennsylvania 15260 